\title{
Implementation of Generalized Discrete Fourier Transform for CDMA on Real Time System
}

\author{
Umesh Sakhare \\ Department of Information \\ Technology \\ G.H. Raisoni College of \\ Engineering, Nagpur
}

\begin{abstract}
This paper deals with simulation of Generalized Discrete Fourier Transform spreading codes used in modulation technique in Code Division Multiple Access on real time system using MATLAB environment. Binary sequences can be used as various spreading code in digital modulation technique. The spreading codes are used to increase the spread spectrum and proper utilization of the spread spectrum in cellular communication system. In CDMA for digital modulation technique various spreading codes like Gold codes, M-ary Binary sequences, Walsh codes and Constant modulus function set (GDFT) are used. These codes can be used in various applications like Orthogonal Frequency Division Multiple Access (OFDM), Discrete Multi-tone and Code Division Multiple Access.

This paper proposes real time system model in which GDFT spreading code modulated with voice or speech as input analog signal. Performance of Generalized Discrete fourier transform with non linear phase is been determined by some factors. The factors are Bit-Error-Rate, Signal-to-Noise ratio and Mean Square Value of auto-correlation and Mean Square Value of Cross-Correlation responsible to maintain quality of services in digital modulation.
\end{abstract}

\section{Keywords}

Spread Spectrum, Generalized Discrete Fourier Transform, Auto-Correlation function, Cross-Cross Correlation function, PN Sequence, CDMA, Gold Codes, Walsh Codes, Discrete Fourier Transform.

\section{INTRODUCTION}

Code Division Multiple Access (CDMA) with Direct Sequences spread spectrum is one of the emerging technologies in Wireless communication. CDMA is famous for better voice quality and better result. CDMA technology uses Direct Sequence Spread Spectrum. DSSP is responsible to increase band spectrum and proper utilization of bandwidth. Increase in band spectrum will improve the interference and convert the narrowband signal into broadband signal. In Communication, spreading code families like Gold codes, Walsh codes, GDFT have been successfully used for asynchronous communication in DS-CDMA system. Several binary spreading code sets are designed to optimized BER and uses even Cross-Correlation function to recover original data, but it is found that along with even crosscorrelation and odd cross-correlation is also very important. Hence Hidenobu Fukumasa, Ryuji Kohno, Hideki Imai proposed a method to design spreading sequences for CDMA. The properties of these sequences are the absolute values of their odd and even correlation function. They discovered a method for Odd cross-correlation by giving a new set of complex pseudo-random noise sequences $(\mathrm{PN})$. This results in lower peaks for both odd and even correlation, which is useful for achieving stable acquisition and increasing CDMA capacity and also reduces co-channel interference and improves BER performance [2][4].

Mukesh Tiwari, Vaishali Patil, Jaikaran Singh, in their work simulated mathematical model of Gold Codes, Walsh Codes and GDFT. Performance of code families observed only on mathematical model on result of BER and SNR. From the result they conclude GDFT provides better and efficient correlation function which can be exploited in optimum way of asynchronous CDMA communication system rather than Gold and Walsh codes [1].

Syed Islam, T. M. Nazmul Huda, determined the performance on the basis of few particular length of Gold codes and Walsh codes. In this, they had checked behavior of Gold and Walsh code families by adding random noise to the system. Correlations are tested on real time system for the codes, which show much better result. Spreading code used for modulation is generated. For a system a pair of $\mathrm{m}$ sequences of length five is considered. It is found possible to generate gold codes using different pairs of $m$ sequences of different lengths which may be further tested for different system. Random noise addition was based on uniform distribution, therefore it can be further tested for different distribution [3].

Ali Akanshu, Handan Agirman-Tosun proposed a mathematical model of Generalized Discrete Fourier Transform (GDFT) which can be used further for modulation process in digital communication. GDFT can be derived by the definition of Discrete Fourier Transform. GDFT provides a unified theoretical framework. It provides foundation to exploits the phase space to improve the correlation property of constant modulus orthogonal set. GDFT having improved correlation over popular digital spreading code like DFT, Gold and Walsh as well as Oppermann families. It is leading to superior communication performance for the scenario considered in this paper [2][5].

\section{DESIGN OF GENERALIZED DISCRETE FOURIER TRANSFORM}

\subsection{Mathematical Preliminaries and Discrete Fourier Transform}

In this paper we are using GDFT framework to generate spreading codes. Complex spreading code represents complex roots of unity. All roots are placed on the unit circle of unity of the complex $\mathrm{z}$ plane [2].

$\mathrm{N}^{\text {th }}$ root of unity is defined as a complex number $\mathrm{z}$ satisfying the polynomial equation expressed as follows.

$z^{N}-1=0 \quad N \in\{1,2,3\}$ 
All primitive Nth roots of unity satisfy the unique summation property of a geometric series expressed as follows:

$$
\sum_{n=0}^{N-1}\left(z_{p}\right)^{n}=\frac{\left(z_{p}\right)^{n}-1}{z_{p}-1}=\left\{\begin{array}{c}
1, N=1 \\
0, N>1
\end{array}\right\} \forall p
$$

Now define a periodic, constant modulus complex sequence $\left\{e_{r}(n)\right\}$ as the $r$ th power of the first primitive Nth roots of unity

$$
e_{r}(n) \triangleq\left(z_{1}^{r}\right)=e^{j(2 \pi r / N) n}
$$

Where $\mathrm{n}=0,1,2,3, \ldots \mathrm{N}-1$ and $\mathrm{r}=0,1,2, . . \mathrm{N}-1$ The complex sequence III over a finite discrete time interval in a geometric series is expressed as follows [1][2]

$$
\left.\begin{array}{l}
\frac{1}{N} \sum_{n=0}^{N-1} e_{r}(n)=\frac{1}{N} \sum_{n=0}^{N-1}\left(z_{1}^{r}\right)^{n} \\
=\frac{1}{N} \sum_{n=0}^{N-1} e^{j(2 \pi r / N) n} \\
=\left\{\begin{array}{cc}
1, & r=m N \\
0, & r \neq m N \\
m=\text { integer }
\end{array}\right.
\end{array}\right\}
$$

Then from equation (4) DFT on set $\left\{e_{r}(n)\right\}$ satisfying orthonormality conditions [2]

$\left\langle e_{k}(n), e_{l}^{*}(n)\right\rangle=\frac{1}{N} \sum_{n=0}^{N-1} e^{j(2 \pi / N)(k-l) n}$

$$
\left\{\begin{array}{cc}
1, & r=k-l=m N \\
0, \quad r=k-l \neq m N \\
m=\text { integer }
\end{array}\right\}
$$

The Notation $(*)$ represents complex conjugates function of a function.

\subsection{Generalized Discrete Fourier Transform}

Generalize (4) by writing the phase difference of two function $\varphi_{k l}(n)=\varphi_{k}(n)-\varphi_{l}(n)=r \forall n$ and expressing constant modulus function set as follows [2],

$\frac{1}{N} \sum_{n=0}^{N-1} e^{j(2 \pi \varphi k(n) / N) n} e^{-j(2 \pi \varphi k(n) / N) n}$

Function for a new set are defined as follows

$e_{k}(n) \triangleq e^{j(2 \pi / N) \varphi_{k(n) n}}$

where $\mathrm{k}=0,1,2, \ldots \ldots, \mathrm{N}-1$

This is orthogonal function set as the Generalized Discrete Fourier Transform (GDFT).

\subsection{Design Methodology of Generalized Discrete Fourier Transform \\ DFT matrix of $\mathrm{N} \times N$ expressed as}

$A_{D F T}=\left[A_{D F T_{(k, n)}}\right]$

$A_{D F T}=\left[e^{j(2 \pi / N) k n}\right] \quad \mathrm{k}, \mathrm{n}=0,1,2, \mathrm{~N}-1$

(7)
Now, we will define a GDFT by relaxing the linear phase property of DFT without compromising the orthogonality. This is a marked departure from the traditional Fourier analysis including DFT where any set regardless continuous or discrete in time has its linear phase functions. Hence, we express the square GDFT matrix as a product of the three orthogonal matrices as follows [2]

$$
\begin{gathered}
A_{G D F T}=G_{1} A_{D F T} G_{2} \\
A_{G D F T} A_{G D F T}^{-1}=I \\
A^{-1}{ }_{G D F T}=A_{G D F T}^{* T} \\
G_{1} G_{1}^{* T}=I \quad G_{2} G_{2}^{* T}=I \\
=I
\end{gathered}
$$

Where G1 and G2 are constant modulus diagonal matrices and written as [2]

$G_{1}(k, n)=\left\{\begin{array}{lr}e^{j \theta_{k k},} & k=n \\ 0, & k \neq n \\ k, n=0,1, \ldots N\end{array}\right\}$

and

$$
G_{1}(k, n)=\left\{\begin{array}{lr}
e^{j \gamma_{n n},} & k=n \\
0, & k \neq n \\
k, n=0,1, \ldots N
\end{array}\right\}
$$

\subsection{Performance Matrices of Generalized Discrete Fourier Transform}

In order to compare performance of code families, several objective performance metrics were used in the literature. All the metrics used in this study depend on a periodic correlation functions (ACF) of the spreading code set. The ACF metric is defined for the complex sequences $\left\{e_{k}(n)\right\}$ and $\left\{e_{l}(n)\right\}$ [8].

$$
\begin{aligned}
& d_{k, l}(m) \\
& =\left\{\begin{array}{c}
\frac{1}{N} \sum_{n=0}^{N-1-m} e_{k}(n) e_{l}^{*}(n+m), 0<m \leq N-1 \\
\frac{1}{N} \sum_{n=0}^{N-1+m} e_{k}(n-m) e_{l}^{*}(n), \quad 1-N<m \leq 0 \\
0, \quad|m| \geq N
\end{array}\right\}
\end{aligned}
$$

Cross correlation and Auto correlation is created by using this matrix. By using mathematical based model result can be determined.

\section{PROPOSED SYSTEM}

The proposed work is the MATLAB simulation of GDFT on a real time system model. Real time system model architecture designed as below 


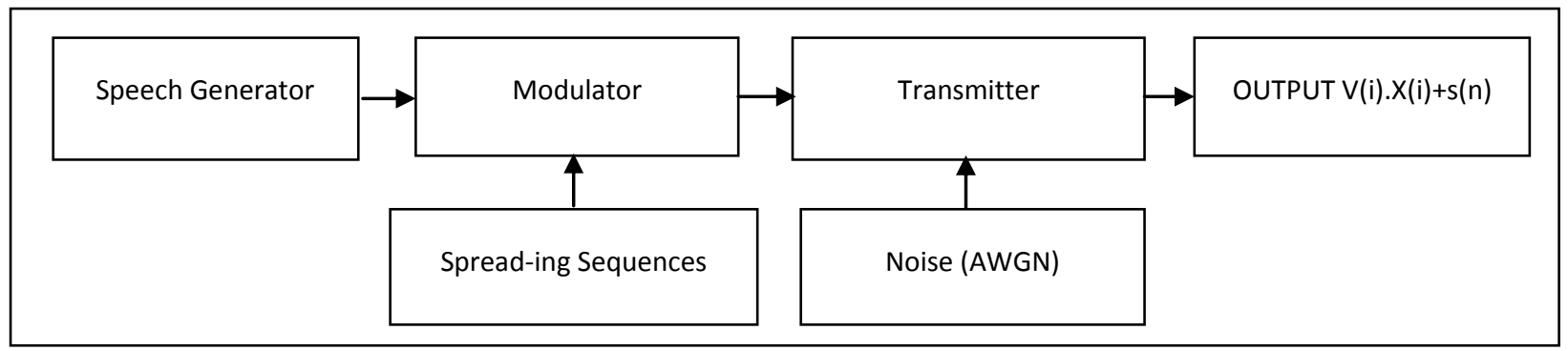

Fig 1 Real Time System Model at Transmitter

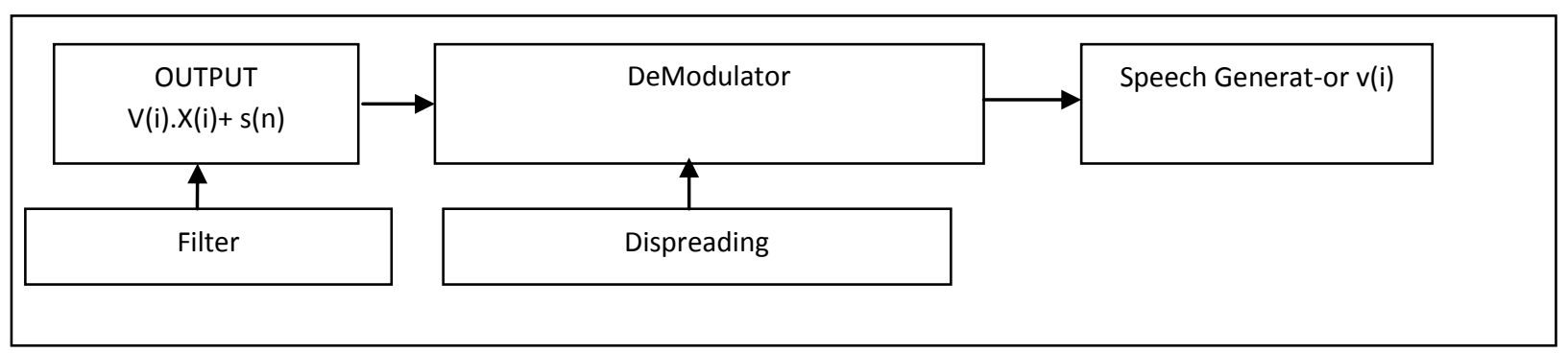

Fig 2 Real Time System at Receiver

In this paper Real Time System work on input voice speech signal V(i) of different person which is digital in nature and mixed with the various spreading codes. The performance of Real Time System can be observed on BER, SNR and Mean Square value. Real Time System approach works on various spreading coding techniques and mentioned in below section.

\subsection{Design of Real Time System Model with Generalized Discrete Fourier \\ Transform}

A constant modulus function set used to design spreading code name as Generalized Discrete Fourier Transform GDFT. Derivation of GDFT already discussed in above section. Literature reviews that GDFT is best spreading code to spread spectrum technique. In the proposed plan we are developing Real Time Model which uses the GDFT spreading codes. The result of this system directly view on real time model on the basis of that we will proposed the best and efficient spreading codes for DSSS techniques.

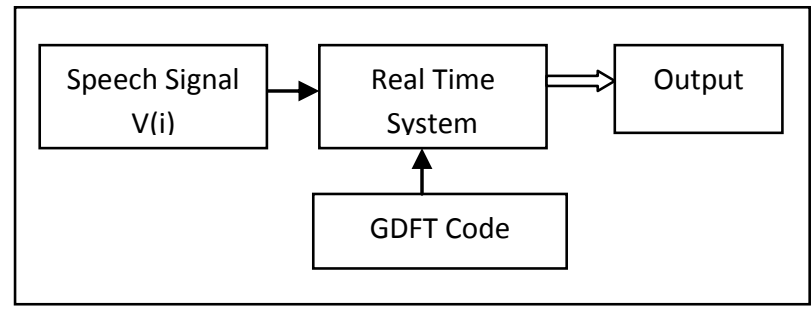

Fig. 3 Design of GDFT Code on RTS

\section{ALGORITHM TO DESIGN GENERALIZED DISCRETE FOURIER TRANSFORM}

Step 1: Find $\mathrm{N} \times \mathrm{N}$ DFT by using

$$
A_{D F T}=\left[e^{j(2 \pi / N) k n}\right]
$$

Step 2: G1, G2 are constant modulus diagonal matrices which can be given by a metrics as

$$
G_{1}(k, n)=\left\{\begin{array}{lr}
e^{j \gamma_{n n},} & k=n \\
0, & k \neq n \\
k, n=0,1, \ldots N
\end{array}\right\}
$$

and

$$
G_{1}(k, n)=\left\{\begin{array}{ll}
e^{j \theta_{k k},} & k=n \\
0, & k \neq n \\
k, n=0,1, \ldots N
\end{array}\right\}
$$

Step 3 : Find GDFT

$$
A_{G D F T}=G_{1} A_{D F T} G_{2}
$$

Step 4: Find out factor associated with communication channel

a) Maximum value of out of phase Auto Correlation (dam)

b) Maximum value of out of phase Cross Correlation $(\mathrm{dcm}) \mathrm{c}$ ) Mean Square Value of Auto Correlation Rac,

d) Mean Square Value of Cross Correlation Rcc

e) Merit Factor.

Step 5: Simulate using AWGN.

Step 6: Plot BER and SNR 


\section{RESULT OF GDFT ON REAL TIME SYSTEM}

Fig. 4 shows er(n) which is the periodic constant modulus sequence and plays an important role in Generalized Discrete Fourier Transform. Periodic Sequence is complex valued as the $\mathrm{r}^{\text {th }}$ power of the first primitive Nth root of unity. Hence it has zeros on the unit circle in Z- plane as shown in fig. 5

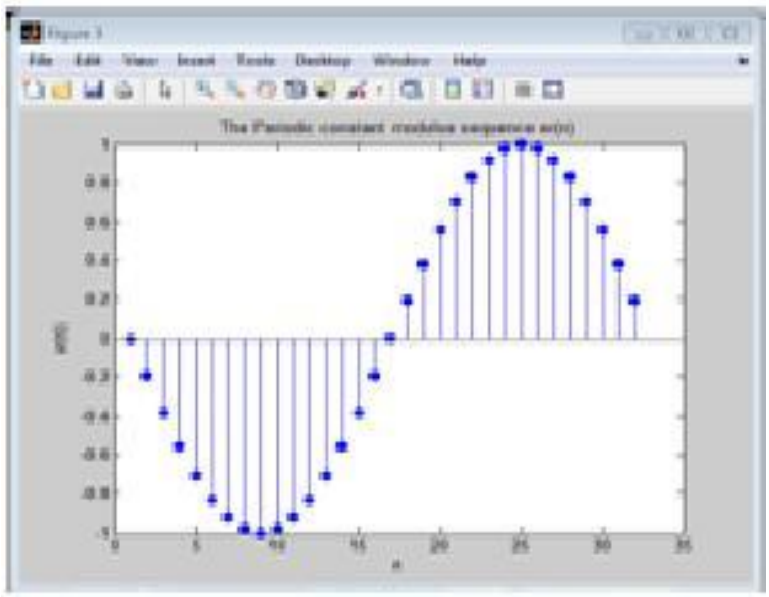

Fig 4 The periodic sequences $e_{r}(n)$ used in GDFT

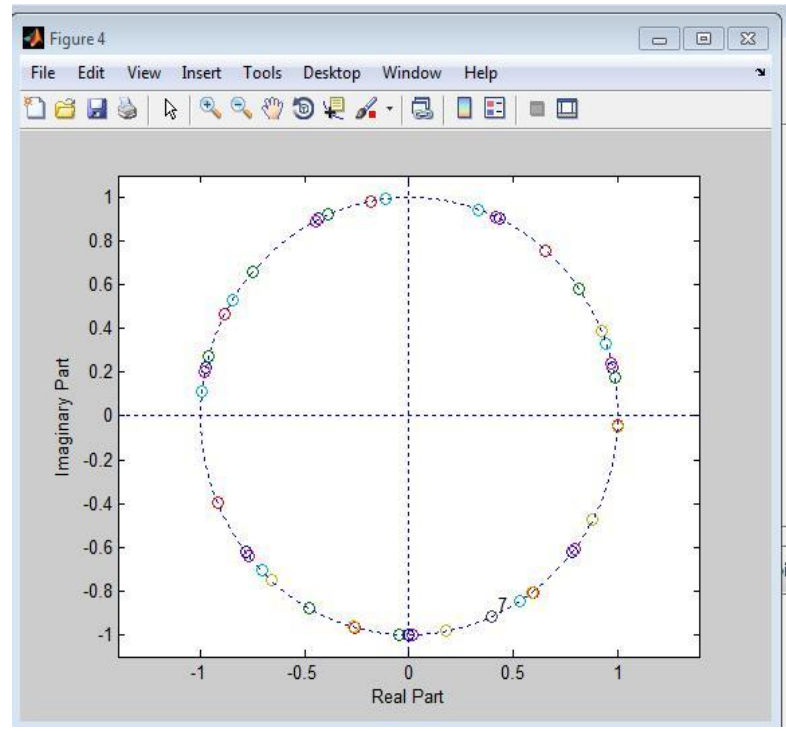

Fig. 5 Pole/Zero plot for primitive Nth root of unit

\subsection{Process of Modulation}

GDFT spreading code is used for the process of modulation. The fig. given below shows the modulation process along with different stages of modulation. First graph shows representation of input signal in time domain. In second graph input signal added with some noise AWGN. Here input signal is having frequency range if 1-500 $\mathrm{HZ}$. Adaptive White Gaussian Noise is noise generated by channel. Adaptive White Gaussian Noise which is random in nature. In above graph red color is indicated as AWGN noise. Third graph shows the 1-31 samples of GDFT spreading code further used in modulation. Fourth graph shows process of modulation with noise and without noise figure. Analog signal generated after modulation process is a high frequency signal. The signal generated In Process of modulation input signal is divided into number of samples. Here, in modulation matrix with row [1-31] samples bit should be multiplied with [1-31] samples bit of GDFT sequence as shown in figure. Samples should be a matrix of sequence 1-31.After completing Modulation process is

$\mathbf{Y}(\mathbf{i})=[\mathrm{V}(\mathbf{i})] \times$ GDFT $].(1 \times 31)$

Y(i) - Output signal generated after modulation.

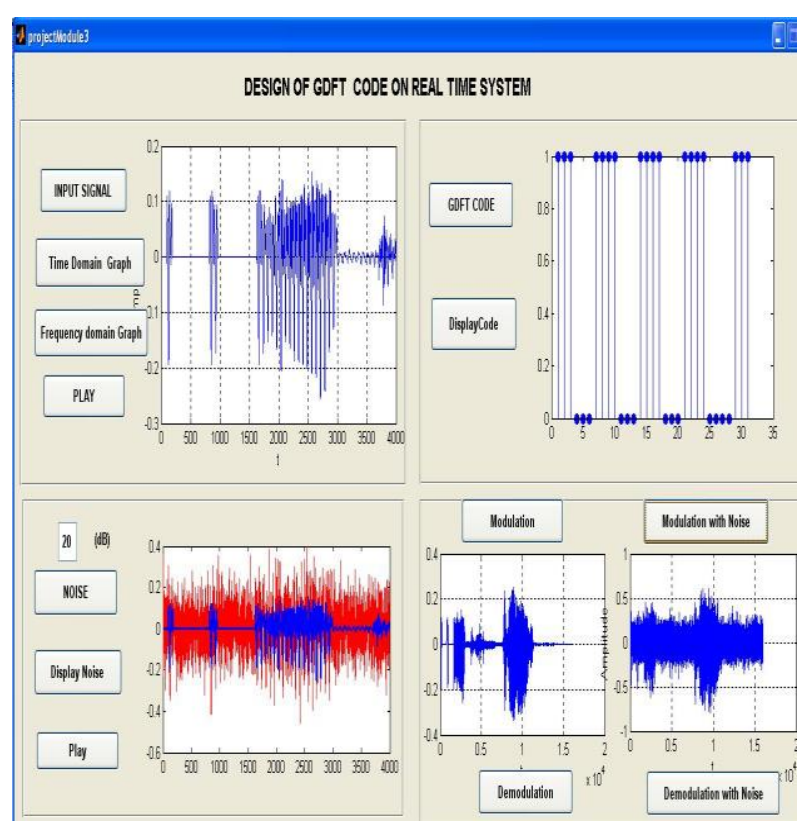

Fig 6 Process of Modulation

\subsection{Process of De-Modulation}

To recover original signal, process of De-Modulation is used, where spreading codes which are to be dispread after transmission is recovered by using the various correlation function. Figure given below shows the process of demodulation, Plot 1 shows the modulated analog signal which is high frequency signal. GDFT code used in modulation is extracted from modulated signal as shown in plot 2. System is having noise which is filtered out by using wide band filter. After process of dispreading, original input signal is recovered which found to be exactly similar to the original input signal with same frequency? Plot 3 shows original analog signal.

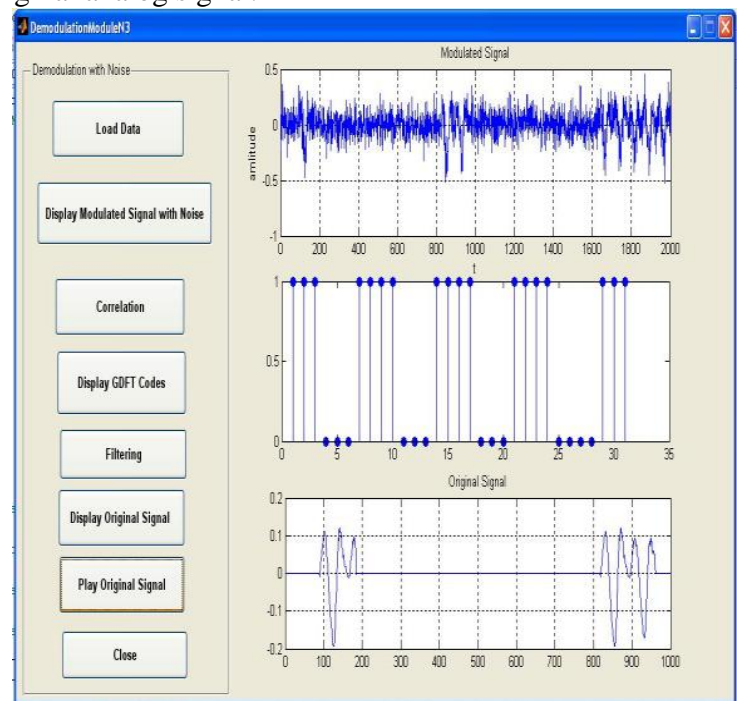

Fig 7 Process of De-Modulation on Real Time System 


\section{PERFORMANCE COMPARISON OF GDFT WITH OTHER SPREADING CODE}

Performance of GDFT code and other families like Gold Code and Walsh code and their correlation is analyzed in terms of Signal to Noise ratio (SNR) and Bit Error Rate. As SNR is increases the BER is decreases. The figure shows the analysis of Gold code, Walsh and GDFT on Real time system. GDFT having better result based on the comparative result as compare to Gold and Walsh code. GDFT is having lower $\mathrm{SNR}$ as BER is increases. Lower cross correlation function plays vital role to improve better result for multipath fading. Figure shows the comparison, in which graph of is below as compare to Gold and Walsh code as Walsh and GDFT on Real time system. Comparison in terms of out of phase Auto correlation (dam), out of phase crosses correlation (dcm), Mean Square value of Auto Correlation $\left(\mathrm{R}_{\mathrm{AC}}\right)$, Mean Square value of cross correlation $\left(\mathrm{R}_{\mathrm{CC}}\right)$.

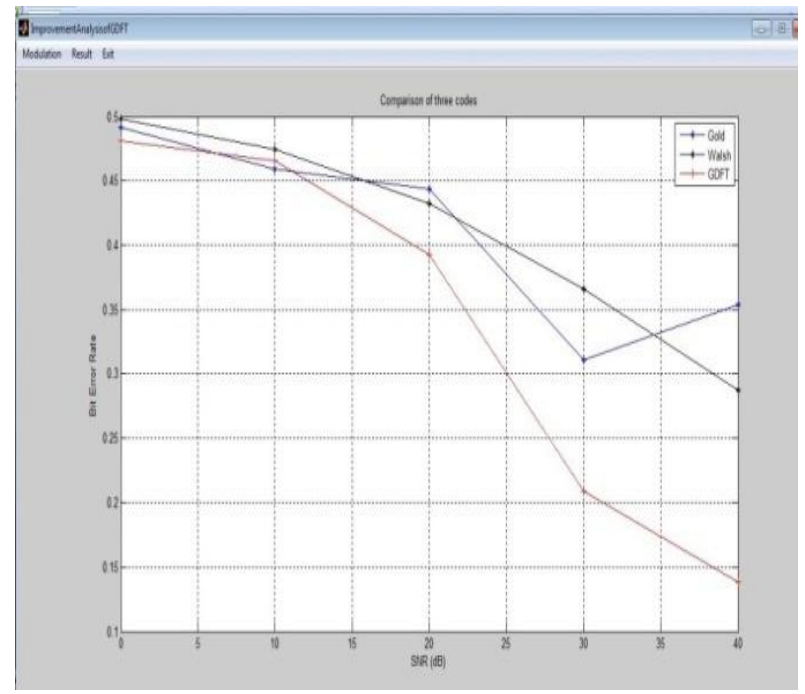

Fig 8 Performance Comparison of GDFT, Walsh and Gold code

Walsh and GDFT on Real time system. GDFT having better result based on the comparative result as compare to Gold and Walsh code. Lower cross correlation function plays vital role to improve better result for multipath fading

Table 1: Comparison Performance of Gold, Walsh and GDFT

\begin{tabular}{|l|l|l|l|l|l|}
\hline Code & dam & Dcm & $\mathbf{R}_{\mathbf{A C}}$ & $\mathbf{R}_{\mathbf{C C}}$ & $\mathbf{F}$ \\
\hline Gold & 1 & 0.0312 & 0.442 & 0.00021 & 2.213867 \\
\hline Walsh & 1 & 0.3605 & 0.127 & 0.00979 & 0.637889 \\
\hline GDFT & 0.342616 & 0.4545 & 0.454 & 0.01414 & 0.006053 \\
\hline
\end{tabular}

\section{CONCLUSION}

In this paper we have implemented spreading code GDFT with non linear phase on Real Time System. GDFT code can be used in CDMA which is having better result and effective correlation function as compare to Gold, Walsh and any other M-Ary binary sequences on real time system. Multipath effect of the channel is affected by low value of Mean Square Value of Auto Correlation. Merit Factor (F) of channel should improved communication path. Merit Factor should be best for CDMA communication. GDFT is responsible to improve uality of services for CDMA. Gold code is best in asynchronous quality of services for CDMA. Gold is best in asynchronous type of data transmission in CDMA. In future, Generalized Discrete Fourier Transform can be implemented in CDMA mobile technology.

\section{REFERENCES}

[1] Vaishali Patil, Jaikaran Singh, Mukesh Tiwari "Simulation of GDFT for CDMA", MPGI National Muti-Conference 2012.

[2] Ali N. Akanshu, Handan Agirman "Generalized Discrete Fourier Transform", IEEE Transaction on Signal Processing, vol 58, NO.9, Septmber 2010.

[3] T. M. Nazmul Huda, Syed Islam "Correlation Analysis of the Gold Codes and Walsh Codes in CDMA Technology”, IEEE, 2009

[4] Hidenobu Fukumasa , Ryuji Kohno, and Hideki Imai "Design of Pseudo Random Noise Sequences with Good Odd and Even Correlation Properties" IEEE Second International Symposius on Spread Sprectrum tech. and Application, Japan Nov 29 Dec 2, 1992.

[5] Richard Haddad Ali Akansu "A new Orthogonal Transform for signal Coding".

[6] Richard Haddad Ali Akansu "A new Orthogonal Transform for signal Coding".

[7] Ian Oppermann "Orthogonal Complex Valued Spreading Sequences with a wide Range of Correlation Properties", IEEE transaction on communication Vol. 45, N0 11, Nov 1997.

[8] A. K. S. Al- Bayati and S. Prasad "Modified constant modulus algorithm for blind DS/CDMA", Electronics Lett, 1999.

[9] Ian Oppermann and B.S. Vucetic "Complex Valued Spreading Sequences with a wide Range of Correlation Properties", IEEE transaction on communication Vol. 45,pp. 365-375 N0 11, March

[10] Cenk Kose, Keith M. Chugg and Thomos R. Halford "Constant Modulus Orthogonal Frequency Division Multiplexing”, 2010 IEEE Military communication conferenece.

[11] Intitive Guide to principles of communications www.complextoreal.co Copy Right 2002 Charan Langton. 